International Journal of Pure and Applied Mathematics

Volume 88 No. 3 2013, 437-442

ISSN: 1311-8080 (printed version); ISSN: 1314-3395 (on-line version)

url: http://www.ijpam.eu

doi: http://dx.doi.org/10.12732/ijpam.v88i3.11

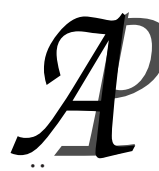

ijpam.eu

\title{
CHARACTERIZATION OF VOLTERRA SPACES
}

\author{
Milan Matejdes \\ Department of Mathematics and Descriptive Geometry \\ Technical University in Zvolen \\ T.G. Masaryka 24, SK-960 53 Zvolen, SLOVAKIA \\ and \\ Department of Mathematics \\ Faculty of Applied Informatics \\ Tomas Bata University in Zlín \\ Nad Stráněmi 4511, CZ-760 05 Zlín, CZECH REPUBLIC
}

\begin{abstract}
In this paper we deal with a characterization of weak Volterra and Volterra spaces which is based on a general concept derived from a given nonempty system $\mathcal{E}$ of nonempty subsets of a topological space. It is shown that a system of all preopen $G_{\delta}$-sets is suitable for characterization of weak Volterra and Volterra spaces.
\end{abstract}

AMS Subject Classification: 54A05, 54E52

Key Words: Volterra space, weakly Volterra space, cluster system, ideal, $\mathcal{J}$-resolvable space, preopen $G_{\delta}$-set, boundary $F_{\sigma}$-set, $\sigma$-nowhere dense set

\section{Introduction}

In the sequel, $X$ is a nonempty topological space. By $\bar{A}, A^{\circ}$ we denote the closure, the interior of $A$ (in space $X$ ), respectively and $\mathcal{U}(z)$ is a base of the open neighborhoods of a point $z$.

By [1], [5] a topological space $X$ is weakly Volterra (Volterra), if for any

Received: July 23, 2013

(c) 2013 Academic Publications, Ltd. url: www.acadpubl.eu 
$G_{\delta}$-sets $A_{1}$ and $A_{2}$ which are dense in $X, A_{1} \cap A_{2}$ is nonempty (dense in $X$ ) and a nonempty open subset $G$ is weakly Volterra (Volterra), if $G$ as a subspace of $X$ is weakly Volterra (Volterra) what is equivalent with condition $A_{1} \cap A_{2} \neq \emptyset$ $\left(A_{1} \cap A_{2}\right.$ is dense in $G$ ) for any $G_{\delta}$-sets (in space $X$ ) $A_{i}$ such that $G \subset \overline{A_{i}}$, $i=1,2$.

Considering so called cluster system the next notions and definitions were introduced in [6].

Definition 1. A nonempty system $\mathcal{E} \subset 2^{X} \backslash\{\emptyset\}$ is called a cluster system. For a set $A$, by $\mathcal{E}(A)$ we denote the set of all points $x \in X$ such that for any $U \in \mathcal{U}(x)$, the intersection $U \cap A$ contains a set form $\mathcal{E}$. A set $A$ is called weakly $\mathcal{E}$-Volterra if for any two sets $A_{1}$ and $A_{2}$, such that $\mathcal{E}(A) \subset \mathcal{E}\left(A_{i}\right)$, $i=1,2, A_{1} \cap A_{2}$ is nonempty and $A$ is $\mathcal{E}$-Volterra if $A \neq \emptyset$ and $A_{1} \cap A_{2}$ is dense in $A$ for any $A_{i}$ for which $\mathcal{E}(A) \subset \mathcal{E}\left(A_{i}\right), i=1,2$. Finally, if any nonempty open subset of a nonempty open set $G$ contains a set from $\mathcal{E}$, then $\mathcal{E}$ is called a $\pi$-network in $G$.

In [1] a few results can be found which have a nice analogy with Banach category theorem. Namely, the union of any family of nonempty open non weakly Volterra subspaces is not weakly Volterra ([1, Lemma 3.5], [5]). Moreover, $X$ is Volterra if and only if any nonempty open subspace is weakly Volterra $[1$, Lemma 3.3]. Similar results hold in $\mathcal{E}$-Volterra and weakly $\mathcal{E}$-Volterra setting.

Theorem 1. (see [6]) Let $\mathcal{E}$ be a $\pi$-network in a nonempty open set $X_{0} \subset X$.

(1) $X_{0}$ is $\mathcal{E}$-Volterra if and only if any nonempty open subset of $X_{0}$ is weakly $\mathcal{E}$-Volterra.

(2) The union of any family of nonempty open non weakly $\mathcal{E}$-Volterra subsets of $X_{0}$ is not weakly $\mathcal{E}$-Volterra.

For further results concerning weakly $\mathcal{E}$-Volterra and $\mathcal{E}$-Volterra spaces see [6].

In [3] the notion of $\mathcal{J}$-resolvability was introduced, where $\mathcal{J}$ is an ideal in $X$. A set $A$ is $\mathcal{J}$-dense if for any nonempty open set $U$ the intersection $A \cap U$ is not from $\mathcal{J}$. A space $X$ is $\mathcal{J}$-resolvable if there are two disjoint subsets of $X$ which are $\mathcal{J}$-dense and $X$ is resolvable if it is $\mathcal{J}$-resolvable, where $\mathcal{J}=\{\emptyset\}$ (i.e., if there are two disjoint dense sets in $X)$. Put $\mathcal{E}_{\mathcal{J}}:=\{A \subset X: A$ is not from $\mathcal{J}\}$. If $X$ is $\mathcal{J}$-resolvable, then $X$ is not weakly $\mathcal{E}_{\mathcal{J}}$-Volterra. The opposite implication holds, provided $\mathcal{J}$ is proper and $\mathcal{E}_{\mathcal{J}}(X)=X$. If $\mathcal{J}=2^{X}$, then $X$ is not $\mathcal{J}$ resolvable and $\mathcal{E}_{\mathcal{J}}=\emptyset$ what is by Definition 1 excluded. If $\mathcal{E}_{\mathcal{J}}(X) \neq X$, then $X$ is not $\mathcal{J}$-resolvable (there is no $\mathcal{J}$-dense set). Consider $X=\left\{0, \frac{1}{2}, \frac{1}{3}, \frac{1}{4}, \ldots\right\}$ 
with the usual topology and $\mathcal{J}=\{A: A$ is finite $\}$. It is clear there are two infinite disjoint sets $A_{1}, A_{2}$ for which $\mathcal{E}_{\mathcal{J}}\left(A_{1}\right)=\mathcal{E}_{\mathcal{J}}\left(A_{2}\right)=\mathcal{E}_{\mathcal{J}}(X)=\{0\}$, so $X$ is not weakly $\mathcal{E}_{\mathcal{J}}$-Volterra.

A set $A$ is called a boundary $F_{\sigma}$-set ( $\sigma$-nowhere dense set, see [2]) if $A^{\circ}=\emptyset$ and $A$ is $F_{\sigma}$. The smallest ideal that contains all boundary $F_{\sigma}$-sets will be denoted by $\mathcal{J}_{\sigma}$. As is showed in [2], if $X$ is $\mathcal{J}_{\sigma}$-resolvable (strong irresolvable, i.e., any nonempty open subspace of $X$ is irresolvable), then $X$ is Volterra. The converse is not true, see [2, Example 3.8]. That means, "Volterraness" is not characterized by the ideal $\mathcal{J}_{\sigma}$ (by $\mathcal{E}_{\mathcal{J}_{\sigma}}$ ). It seems the notion of $\mathcal{J}$-dense set stated above by condition $A \cap U \notin \mathcal{J}$ (equivalently $A \cap U \in \mathcal{E}_{\mathcal{J}}$ ) for any nonempty open set $U$ is not adequate. A cluster system setting is more flexible and a suitable specification of $\mathcal{E}$ allows to characterize "Volterraness". Replacing the system $\mathcal{E}_{\mathcal{J}_{\sigma}}$ by a system of all sets which are preopen and $G_{\delta}$, we can obtain a characterization of weakly Volterra and Volterra spaces as we will see in the next section.

\section{Weakly Volterra and Volterra Spaces}

In this paragraph we will deal with a special case of a cluster system which characterizes weakly Volterra and Volterra spaces. Consider a cluster system $\mathcal{E}_{\delta}^{p}$ of all sets which are nonempty preopen and $G_{\delta}$ (a set $A$ is preopen if $A \subset(\bar{A})^{\circ}$, see [4]). More precisely, $A \in \mathcal{E}_{\delta}^{p}$ (we will refer $A$ as a preopen $G_{\delta}$-set) if and only if it is $G_{\delta}$ and there is a nonempty open set $G \supset A$ such that $A$ is dense in $G$ or equivalently, $A$ is $G_{\delta}$ and there is a nonempty open set $G \supset A$ such that $G \backslash A$ is a subset of some boundary $F_{\sigma}$-set (namely $G \backslash A \subset \bar{G} \cap(X \backslash A)$ ). Evidently, $\mathcal{E}_{\delta}^{p}$ is not an ideal, but it is closed with respect to finite union.

Lemma 1. Let $\left\{A_{t}\right\}_{t \in T}$ be a family of $G_{\delta}$-sets and $\left\{G_{t}\right\}_{t \in T}$ be a family of nonempty open pairwise disjoint sets such that $A_{t} \subset G_{t}$. Them $\cup_{t \in T} A_{t}$ is a $G_{\delta}$-set.

Proof. Let $A_{t}=\cap_{n=1}^{\infty} H_{t}^{n}, H_{t}^{n} \subset G_{t}$ and $H_{t}^{n}$ be open. Since $G_{t} \cap G_{s}=\emptyset$ for any $t \neq s, \cup_{t \in T} A_{t}=\cap_{n=1}^{\infty}\left(\cup_{t \in T} H_{t}^{n}\right)$ that is a $G_{\delta^{-}}$-set.

Theorem 2. A topological space $X$ is Volterra if and only if any nonempty open subset of $X$ is weakly $\mathcal{E}_{\delta}^{p}$-Volterra.

Proof. " $\Rightarrow$ " Note that $\mathcal{E}_{\delta}^{p}$ is a $\pi$-network in any nonempty open set $G$, so $\mathcal{E}_{\delta}^{p}(G)=\bar{G}$. 
Let $X$ be Volterra and $G$ be nonempty open. Suppose $G$ is not weakly $\mathcal{E}_{\delta}^{p}$-Volterra. Then there are two sets $A, B$ such that $\mathcal{E}_{\delta}^{p}(A) \supset \mathcal{E}_{\delta}^{p}(G), \mathcal{E}_{\delta}^{p}(B) \supset$ $\mathcal{E}_{\delta}^{p}(G)$ and $A \cap B=\emptyset$. Since $\mathcal{E}_{\delta}^{p}(A) \supset \mathcal{E}_{\delta}^{p}(G)=\bar{G} \supset G$, there is $A_{0}$ from $\mathcal{E}_{\delta}^{p}$ and $A_{0} \subset G \cap A$. Further, there is a nonempty open set $G_{0}$ (we can suppose $\left.G_{0} \subset G\right)$ such that $A_{0} \subset G_{0}$ and $A_{0}$ is dense in $G_{0}$.

Since $\mathcal{E}_{\delta}^{p}(B) \supset \mathcal{E}_{\delta}^{p}(G)=\bar{G} \supset G_{0}$, there is $B_{0}$ from $\mathcal{E}_{\delta}^{p}$ and $B_{0} \subset G_{0} \cap B$. Further there is a nonempty open set $B_{1}$ (we can suppose $B_{1} \subset G_{0}$ ) such that $B_{0} \subset B_{1}$ and $B_{0}$ is dense in $B_{1}$. Note $A_{0} \cap B_{0}=\emptyset\left(A_{0} \cap B_{0} \subset A \cap B=\emptyset\right)$.

Since $A_{0}$ and $B_{0}$ are $G_{\delta}$ and dense in $B_{1}, A_{1}:=\left(X \backslash \bar{B}_{1}\right) \cup\left(A_{0} \cap B_{1}\right)$ and $A_{2}:=\left(X \backslash \bar{B}_{1}\right) \cup B_{0}$ are dense in $X$ and $G_{\delta}$. Since $X$ is Volterra, then $A_{1} \cap A_{2}=X \backslash \bar{B}_{1}$ is dense in $X$, contradiction.

$" \Leftarrow "$. Suppose any nonempty open subset of $X$ is weakly $\mathcal{E}_{\delta}^{p}$-Volterra and $A, B$ are $G_{\delta}$ and dense in $X$, that means $\mathcal{E}_{\delta}^{p}(A)=\mathcal{E}_{\delta}^{p}(B)=X$. Let $G$ be a nonempty open set. Then $A \cap G, B \cap G$ are $G_{\delta}$ dense in $G$, so $\mathcal{E}_{\delta}^{p}(A \cap G) \supset \mathcal{E}_{\delta}^{p}(G)$ and $\mathcal{E}_{\delta}^{p}(B \cap G) \supset \mathcal{E}_{\delta}^{p}(G)$. Since $G$ is weakly $\mathcal{E}_{\delta}^{p}$-Volterra, $(A \cap G) \cap(B \cap G)=$ $A \cap B \cap G \neq \emptyset$. So $A \cap B$ is dense in $X$, that means $X$ is Voltera.

The next corollary follows from Theorem 2 and Theorem 1 item (1).

Corollary 1. A topological space $X$ is Volterra if and only if $X$ is $\mathcal{E}_{\delta}^{p}$ Volterra.

Theorem 3. A nonempty open subset $G$ of $X$ is weakly Volterra if and only if $G$ is weakly $\mathcal{E}_{\delta}^{p}$-Volterra.

Proof. " $\Rightarrow$ " Let $G$ be nonempty open weakly Volterra and $A^{1}, A^{2}$ be such that $\mathcal{E}_{\delta}^{p}\left(A^{i}\right) \supset G, i=1,2$. Suppose contrary $A^{1} \cap A^{2}=\emptyset$.

From inclusion $\mathcal{E}_{\delta}^{p}\left(A^{i}\right) \supset G$ and from Zorn lemma there is a family $\left\{G_{t}\right\}_{t \in T}$ of pairwise disjoint nonempty open subsets of $G$ and two families $\left\{A_{t}^{i}\right\}_{t \in T}(i=$ $1,2)$ of sets from $\mathcal{E}_{\delta}^{p}$ such that for $i=1,2$

(1) $\cup_{t \in T} G_{t}$ is dense in $G$,

(2) $A_{t}^{i} \subset G_{t} \cap A^{i}$ and $A_{t}^{i}$ is dense in $G_{t}$.

Put $C^{i}=\cup_{t \in T} A_{t}^{i}, i=1,2$. Since $A^{1} \cap A^{2}=\emptyset$ and $G_{t} \cap G_{s}=\emptyset(t \neq s)$, $C^{1} \cap C^{2}=\emptyset$. Moreover, $C^{i}$ is dense in $\cup_{t \in T} G_{t}$ and by item (1), $C^{i}$ is dense in $G, i=1,2$. By Lemma $1, C^{i}$ is a $G_{\delta^{-}}$set and since $G$ is weakly Voltera, $\emptyset \neq C^{1} \cap C^{2}$, a contradiction.

The opposite implication is clear. 
The next corollary follows from Theorem 2 and Theorem 3 .

Corollary 2. (see [1, Lemma 3.3]) A topological space $X$ is Volterra if and only if any its nonempty open set is weakly Volterra.

Theorem 4. A nonempty open set $G$ is not weakly Volterra if and only if $G \subset P \cup R$ where $P, R$ are boundary $F_{\sigma}$-sets.

Proof. " $\Rightarrow$ "Since $G$ is not weakly Volterra, their are two disjoint $G_{\delta^{-}}$ subsets $G_{1}, G_{2}$ of $G$ which are dense in $G$. Then $G \subset\left[\bar{G} \cap\left(X \backslash G_{1}\right)\right] \cup[\bar{G} \cap$ $\left.\left(X \backslash G_{2}\right)\right]$. We will show that $A_{i}:=\bar{G} \cap\left(X \backslash G_{i}\right)$ has empty interior, $i=1,2$. If not, there is a nonempty open set $H \subset \bar{G} \cap\left(X \backslash G_{i}\right)$. Since $H_{0}:=H \cap G \neq \emptyset$ and $H_{0} \cap G_{i}=\emptyset$, we have a contradiction with the assumption that $G_{i}$ is dense in $G$. So $P:=\left[\bar{G} \cap\left(X \backslash G_{1}\right)\right]$ and $R:=\left[\bar{G} \cap\left(X \backslash G_{2}\right)\right]$ are boundary $F_{\sigma}$-sets and $G \subset P \cup R$.

$" \Leftarrow$ " If $G \subset P \cup R$ and $P, R$ are boundary $F_{\sigma}$-sets, then $[G \cap(X \backslash P)]$ and $[G \cap(X \backslash R)]$ are disjoint $G_{\delta}$-subsets of $G$ which are dense in $G$, so $G$ is not weakly Volterra.

Corollary 3. A topological space $X$ is weakly Volterra (Volterra) if and only if $X$ (any nonempty open set $G \subset X$ ) is not (a subset of) the union of two boundary $F_{\sigma}$-sets.

By [2, Theorem 2.5], any subset of $X$ that is union of finite many $\sigma$-nowhere dense sets (i.e., boundary $F_{\sigma}$-sets) can be expressed as the union of exactly two $\sigma$-nowhere dense sets (two boundary $F_{\sigma}$-sets). So we have the following ([2, Proposition 3.3 and Lemma 3.4], [5]).

Corollary 4. A topological space $X$ is weakly Volterra (Volterra) if and only if $X \notin \mathcal{J}_{\sigma}\left(G \notin \mathcal{J}_{\sigma}\right.$ for any nonempty open set $\left.G \subset X\right)$.

\section{Acknowledgments}

Partially supported by APVV-0269-11.

\section{References}

[1] J. Cao, D. Gauld, Volterra spaces revisited, J. Aust. Math. Soc., 79 (2005), 61-76, doi: 10.1017/S1446788700009332.

[2] J. Cao, S. Greenwood, The ideal generated by $\sigma$-nowhere dense sets, $A p$ plied General Topology, 7(2) (2006), 253-264. 
[3] J. Dontchev, M. Ganster, D. Rose, Ideal Resolvability, Topology Appl., 93 (1999), 1-16, doi: 10.1016/S0166-8641(97)00257-5.

[4] M. Ganster, Preopen sets and resolvable spaces, Kyungpook Math. J., 27 (1987), 135-143.

[5] D. Gauld, S. Greenwood, Z. Piotrowski, On Volterra spaces III:topological operations, Topology Proc., 23 (1998), 167-182.

[6] M. Matejdes, Generalized Volterra spaces, Int. J. Pure Appl. Math., 85 (2013), 955-963, doi: 10.12732/ijpam.v85i5.13. 